\title{
Discussion
}

Buscombe: Why was IC 2602 selected if its radial velocity is not well known? Two other nearby southern clusters, IC 2391 and M7, contain rather many peculiar A stars.

Eggen: Proper motions are not well known for the last two clusters.

Blaauw: In determining distances and luminosities, Dr. Eggen assumed a constant value of the velocity component $V$ for the stars involved. This was justified by the introductory remarks about the natural selection of stars of ages of $5 \times 10^{8} \mathrm{yr}$ in the solar neighbourhood, these stars having been formed in a region $<500$ pc. What justification is there in applying this theory to the $\mathrm{B}$ stars which are 10 times younger?

Eggen: The point is that at least those with $V=-27$ are members of the association; others may also belong but because of the dispersion in $V$ they will not be picked up.

\section{SPACE DISTRIBUTION OF 156 GALACTIC STAR CLUSTERS AND OF 61 HII REGIONS*}

\author{
W. BECKER
}

Astronomische Anstalt, Basel-Binningen

The galactic star clusters are today the only objects whose individual distances and ages can be determined rather precisely. Therefore the space distribution as a function of time can be studied. In case they form a spiral structure, the lifetime of such a structure can be studied too.

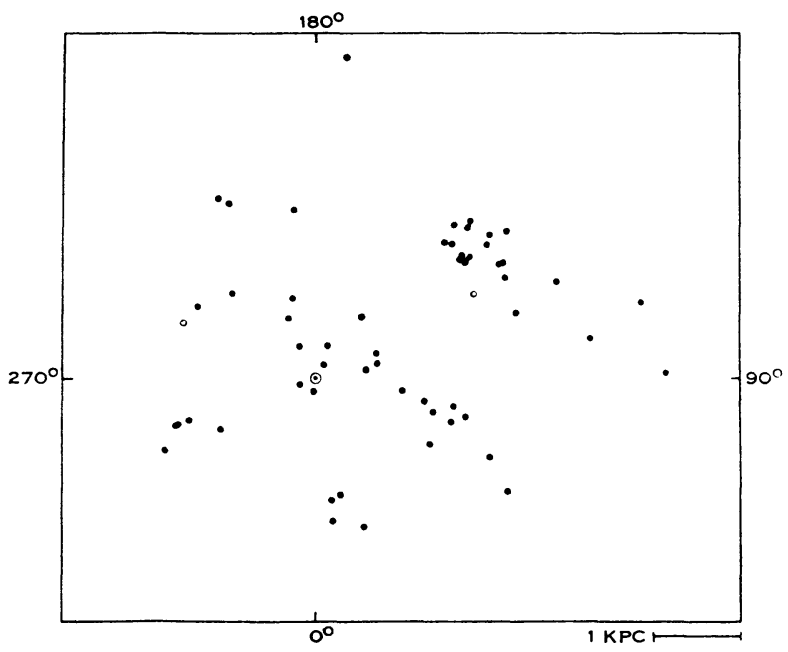

Fig. 1.-Distribution of clusters with earliest spectral type between $\mathrm{O}$ and $\mathrm{B2}$.

In the following, we rely exclusively on distances obtained by the method of three-colour photometry. As this method is used in two different ways, all distances are reduced to a homogeneous system applying the method developed at Basel-

* Published in detail in Zs. f. Ap. 56: 257-63 and 57: 117-34. 
Binningen. This means that for the determination of the distances, the two colourmagnitude diagrams are used (the two-colour diagram is not used) and also the assumption is made that the reddening is uniform over the area of a cluster.

The principal sources for the catalogue of the distances of 156 galactic star clusters are the important paper of Hoag et al. (1961) containing the observational results for 69 clusters and an earlier paper of the author (1961) giving the data for 82 clusters collected from different publications.

The distribution of the clusters with earliest spectral type between B3 and F in the galactic plane does not show an indication of a particular structure. The distribution seems to be random. On the contrary, the distribution of the clusters with earliest spectral type between $\mathrm{O}$ and $\mathrm{B} 2$ follows three spiral arms which are clearly seen (Fig. 1). The spiral arm on whose inner side the Sun lies extends from $l \mathrm{II}=50^{\circ}$ to $l \mathrm{II}=230^{\circ}$; the outer arm (Perseus-Cassiopeia) and the next inner arm are more or less parallel to it.

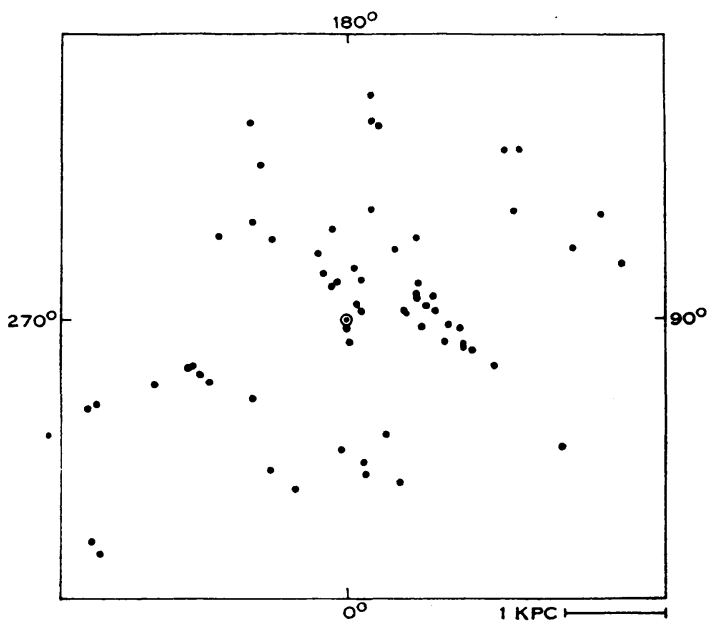

Fig. 2.-Distribution of HII regions in the galactic plane.

The distance of the clusters from the galactic plane does not depend on the distance from the Sun, indicating that these distances are not affected by a scale error. On the average, the distance of the clusters from the galactic plane is approximately 60 pc. It does not depend much on the earliest spectral type and not on the spiral arm to which a cluster belongs.

The distances of $47 \mathrm{HII}$ regions are determined by using three-colour photometry for 10 galactic star clusters and $\mathrm{H}_{\gamma}$ luminosities and photoelectric colour indices for 45 stars in 37 HII regions which are responsible for the HII emission. These data were taken mostly from catalogues published by R. M. Petrie, A. Beer, I. M. Kopylov, and W. A. Hiltner. The distances of 14 HII regions were taken from the list given by Morgan, Sharpless, and Osterbrock. The catalogue of distances of HII regions comprises this way 61 objects.

The distribution of these objects in the galactic plane is given in Figure 2. There seems to be a strong indication that the HII regions follow three spiral arms, which 
are exactly the same ones as for galactic star clusters of early type. The scattering is larger for the HII regions because the accuracy of the distances is lower than for clusters.

The three spiral arms shown by the young star clusters and by the HII regions do not coincide with the spiral arms shown by the HI clouds.

\section{References}

Becker, W. (1961).-Zs. f. Ap. 51 : 151-62.

Hoag, A. A., Johnson, H. L., Iriarte, B., Mitcheli, R. I., Halla., K. L., and Sharpless, S. (1961).-Publ. U.S. Naval Obs. 17: 345-542.

\section{Discussion}

Bok: The distances for galactic clusters in the range $260^{\circ}<l^{\mathrm{II}}<270^{\circ}$ and $300^{\circ}<l^{\mathrm{II}}<325^{\circ}$ can be checked with the aid of radial velocities for $m=9$ to 12 . The gaps will be filled in shortly. Combining results from luminosity classifications and $\mathrm{H} \beta$ absolute magnitudes will provide anchor points in distance for the interpretation of radial velocities and the reflex of galactic rotation.

Perek: Ruprecht has determined distances of a number of faint early-type clusters. (Slide shown.) The spiral structure derived by Professor Becker was confirmed.

Kerr : The diagram comparing cluster and hydrogen spiral arms is hard to understand, as the published hydrogen diagrams show arms that are much more circular in form.

Westerhout : Dr. Becker used an artist's impression to compare the cluster distribution with that of neutral hydrogen whereas an actual contour diagram would have been the correct one to use. Also one should realize that in the neighbourhood of the Sun the method used in the $21 . \mathrm{cm}$ line work for determining distance from radial velocity is extremely uncertain: small deviations from circular motion give very large deviations in distance.

Regarding Professor Bok's remark about the work with the nebular spectrograph, one should bear in mind that at those places where the velocity model does not have much influence his combination of photometric and radial velocity observations will not be of much use in giving anchor points for the neutral hydrogen distribution.

Arp: In Professor Becker's final slide he showed a disagreement between the hydrogen arms and the galactic cluster arms in the anticentre region. Regardless of the distance of the HI material at that longitude it was shown at a longitude where there is a break in distribution of clusters. Would one of the radio astronomers present state whether the HI material is indeed observed primarily at this longitude or whether the discrepancy does not exist as was implied by several of the foregoing remarks?

Kerr: This question can be discussed in more detail tomorrow.

\section{STUDIES OF NGC 6067 AND IC 2944}

\section{A. D. Thackeray \\ Radcliffe Observatory}

NGC 6067 is a very rich cluster free of nebulosity in the Norma Cloud, whose colour-magnitude array has been published by Thackeray, Wesselink, and Harding (1962). The choice of stars for measurement was highly restricted to a square approximately $10^{\prime} \times 10^{\prime}$ of arc near the centre, so that above $B=13.0$ only $10 \%$ are expected to be field stars, and between $B=15 \cdot 0$ and $16 \cdot 0$ some $20-30 \%$.

The radial velocity is $-43 \mathrm{~km} / \mathrm{sec}$ and this forms a useful criterion for membership among the stars bright enough for spectroscopy; spectra of 30 stars are available. 